\title{
IMPLEMENTATION OF THE DEVELOPMENT OF A FILTERING ALGORITHM TO IMPROVE THE SYSTEM OF HEARING IN HEARING IMPAIRED WITH COCHLEAR IMPLANT
}

\author{
Salaheddine Derouiche ${ }^{\# 1}$ and Bachir Djedou ${ }^{\# 2}$ \\ "Laboratory of study, research in Instrumentation and Communication of \\ Annaba. \\ University Badji Mokhtar, BP 12, Annaba, 23000 Algeria. \\ ${ }^{1}$ der-salah@hotmail.fr, ${ }^{2}$ djedoubachirdyahoo.fr
}

\begin{abstract}
In this paper, we present the implemented denoising section in the coding strategy of cochlear implants, the technique used is the technique of wavelet bionic BWT (Bionic Wavelet Transform). We have implemented the algorithm for denoising Raise the speech signal by the hybrid method BWT in the FPGA (Field Programmable Gate Array), Xilinx (Virtex5 $X C 5 V L X 110 T)$. In our study, we considered the following: at the beginning, we present how to demonstrate features of this technique. We present an algorithm implementation we proposed, we present simulation results and the performance of this technique in terms of improvement of the SNR (Signal to Noise Ratio). The proposed implementations are realized in VHDL (Very high speed integrated circuits Hardware Description Language). Different algorithms for speech processing, including CIS (Continuous Interleaved Sampling) have been implemented the strategy in this processor and tested successfully.
\end{abstract}

\section{KEYWORDS}

BWT implementation, CIS Strategy, cochlear implant, FPGA, speech processing \& FIR digital filter.

\section{INTRODUCTION}

J. Yao and Y.T. Zhang introduced in their research work [1, 2], Bionic wavelet transform (BWT) as adaptive wavelet transform. It is specially built to model the human auditory system [3]. The BWT is based on the model Giguere-Woodland auditory system [4], a model incorporating feedback electro-active auditory canal, middle ear and cochlea [3]. The term" Bionic" means that a mechanism guided by biologically active [5]. The BWT differs from the conventional Wavelet Transform (WT) in that the time-frequency resolution by the BWT completed, can be adaptively adjusted not only by the frequency variations of the signal, but also by the amplitude instantaneous signal and the derivative of order $1[5,1]$. It is through the mother wavelet, the wavelet transform classical adaptive whereas the BWT is the active control mechanism in the human auditory model that adjusts according to the mother wavelet signal analysis [5 ]. Primarily, the basic idea of the BWT is inspired by the fact that we want to make the shell of the mother 
wavelet time-varying according to the characteristics of the signal and this by introducing a function $T(a, \tau)$ variable in time, in the expression of the mother wavelet $\varphi$ according to the equation $1[5]$.

$$
\varphi(t)=\frac{1}{T(a, \tau) \sqrt{a}} \tilde{\varphi}\left(\frac{t}{T(a, \tau)}\right) \cdot \exp \left(j \omega_{0} t\right)
$$

with $\omega_{0}$ denotes the fundamental frequency of the mother wavelet.

In practice $\omega_{0}$ is equal to 15165.4 for the human auditory system [2]. In the formula (1), the role of the first factor $T(a, \tau)$ multiplying $\sqrt{a}$ is to ensure that energy remains the same for each mother wavelet and the second factor $T(a, \tau)$ is to adjust the casing of the mother wavelet, $\varphi(t)$ without adjusting the center frequency [3]. Thus the adaptive nature of the BWT is captured by the time varying factor, $T(a, \tau)$ representing the scaling of the quality factor of the filter banks of the cochlear, $Q_{e q}$ in each scale over time. Incorporating this directly to the scale factor of the Morlet wavelet, we obtain:

$$
X_{B W T}(a, \tau)=\frac{1}{T(a, \tau) \sqrt{a}} \int x(t) \cdot \widetilde{\varphi}^{*}\left(\frac{t-\tau}{T(a, \tau) \cdot a}\right) \cdot e^{-j \omega_{0}\left(\frac{t-\tau}{a}\right)} d t
$$

where $\tilde{\varphi}$ is the amplitude envelope of the mother wavelet Morlet which is given by:

$$
\widetilde{\varphi}(t)=\exp \left[-\left(\frac{t}{T_{0}}\right)^{2}\right]
$$

with $T_{0}$ is the initial time length of the carrier.

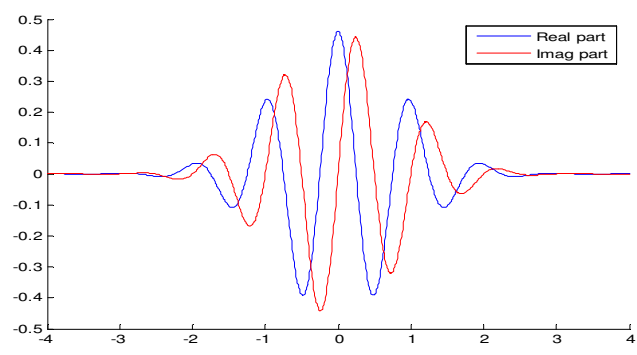

Figure 1. The Morlet wavelet

The discretization of the scale variable a is performed by using the logarithmically spaced across the predefined frequency range desired, such that the center frequency for each scale, is given by equation (4) [3].

$$
\hat{D}_{a p p / d t l}(\omega) \quad \text { With } \mathrm{m}=0,1,2, \ldots
$$

For this implementation, based on the original work of Yao and Zhang cochlear implant for the" coding" [9], the coefficients in 22 scales, $m=7, \ldots, 28$, are calculated using the integration of digital continuous wavelet transform [3]. These 22 scales are logarithmically spaced center frequencies of $225 \mathrm{~Hz}$ to $5300 \mathrm{~Hz}$. The adjustment factor $T(a, \tau)$ for each scale and time is calculated using the equation of adjustment $[5,3]$ : 


$$
T(a, \tau)=\frac{1}{\left(1-G_{1} \frac{C_{s}}{C_{s}+\left|X_{B W T}(a, \tau)\right|}\right) \cdot\left(1+G_{2} \cdot\left|\frac{\partial}{\partial t} X_{B W T}(a, \tau)\right|\right)}
$$

Where $G_{1}$ and $G_{2}$ are active gain factor discussed in $[5,3] . C_{s}$ is a constant representing the effects of nonlinear saturation in the model cochlea $[2,3]$.

The values of $\mathrm{G}_{1}, \mathrm{G}_{2}$ and $\mathrm{C}_{\mathrm{s}}$, dependent on the properties of the target signal and are respectively equal to $0.87,45,0.8$ according to reference [2]. In practice, the partial derivative in equation (5) is approximated using the first difference of the previous points of BWT at this scale [3]. From equation (2), we can see that the adaptation factor $T(a, \tau)$ has an influence on the duration of the amplitude envelope of the wavelet but admits no influence on the frequency of the complex exponential associated. Thus, the BWT can be seen as a mechanism for adapting the time support of the wavelet by quality factor $Q_{e q}$ of the filter model and this cochlear corresponding to each scale [3]. It has been demonstrated [11] that the wavelet coefficients obtained by application of the BWT, $X_{B W T}(a, \tau)$ may beings deducted by multiplying the wavelet coefficients, $X_{C W T}(a, \tau)$ by a constant $K(a, \tau)$ which is a function of the adaptation factor $T(a, \tau)$. For the Morlet wavelet, it is adaptive scaling factor expressed by equation 6 .

$$
K(a, \tau)=\frac{\sqrt{\pi}}{C} \frac{T_{0}}{\sqrt{1+T^{2}(a, \tau)}}
$$

Where $\mathrm{C}$ is a normalization constant calculated from the integral of the square of the mother wavelet [3].

The relationship thus obtained between the $X_{B W T}(a, \tau)$ and $X_{B W T}(a, \tau)$ is given by equation (7).

$$
X_{B W T}(a, \tau)=K(a, \tau) \cdot X_{C W T}(a, \tau)
$$

This representation reduces to a computationally efficient method for calculating wavelet coefficients directly from the bionic wavelet coefficients obtained by the application of the Continuous Wavelet Transform (CWT). This without resorting to numerical integration of equation (2) at each scale [3]. There exist various distinctions between the discretized CWT used for the BWT transform and wavelet packet based on the notion of filter bank and using an orthogonal wavelet as Daubechies family. These differences include the perfect reconstruction in the use of wavelet packet transform; however the discretized CWT is an approximation whose accuracy is dependent on the number and placement of selected frequency bands. Another difference lies in the fact that the Morlet mother wavelet consists of a single frequency with a support decaying time exponentially; however support frequency orthonormal wavelet families used for the dyadic wavelet transform, DWT and the wavelet packet transform, WPT, covers a wider band [3]. Thus the Morlet wavelet is more concentrated frequentially throughout each level, which allowed a direct adaptation of temporal support with minimal impact on the support frequency, central mechanism of adaptation of the BWT [3]. The rest of this paper is organized as follows. In Section 2 and 3, we describe the Algorithm of noise reduction by bionic wavelet transform. In Section 4, we describe the main design of bionic wavelet transform in VHDL. In Section 5 and 6, we show the simulation of the new implementation of BWT denoising algorithm. And conclude the paper by discussing open problems and research challenges in Section 7. 


\section{Algorithm of Noise Reduction by Bionic Wavelet Transform}

Technical noise reduction by bionic wavelet transform, BWT, is summarized by the block diagram is given in Figure $2[5,1]$.

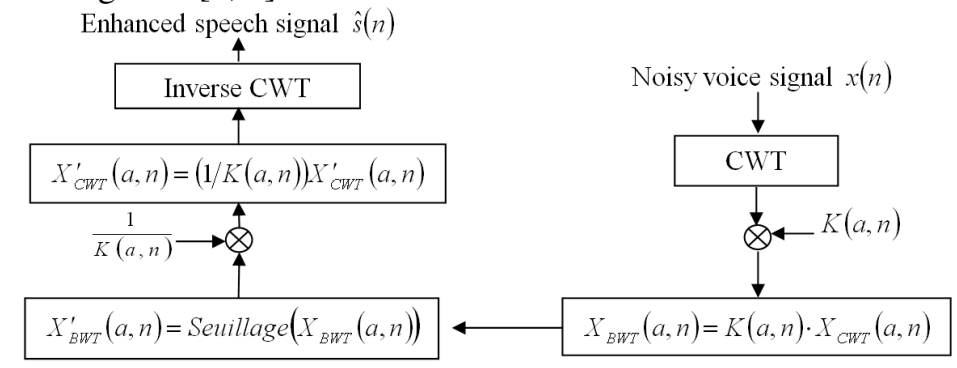

Figure 2. The block diagram of the algorithm for speech enhancement by BWT

The continuous wavelet coefficients are calculated using discrete convolutions in each of the 22 scales using continuous wavelet transform, CWT:

$$
X_{C W T}(a, \tau)=\left\langle x(t), \varphi_{a, \tau}(t)\right\rangle=\frac{1}{\sqrt{a}} \int x(t) \cdot \varphi^{*}\left(\frac{t-\tau}{a}\right) d t
$$

The equations (5) and (6) are used to calculate the factor $\mathrm{K}$ that represents the adjustment of the time support of the wavelet coefficients obtained by application of the continuous wavelet transform, CWT.

In their approach, Michael T. et al [3] used the safe method [15] for calculating the threshold:

$$
\lambda=\underset{0 \leq \lambda \leq \sigma \sqrt{2 \cdot \log n}}{\arg \min }\left\{\hat{\sigma}^{2} n+\sum_{k=1}^{n}\left[\min \left(x(k), \lambda^{2}\right)-2 \hat{\sigma}^{2} I(|x(k)| \leq \lambda)\right]\right\}
$$

Where I denotes the indicator function.

$$
S_{\text {doux }}^{\lambda}\left(d_{l, k}\right)=\operatorname{signe}\left(d_{l, k}\right)\left(\left|d_{l, k}\right|-\lambda\right)_{+} \text {avec } \alpha_{+}=\sup (\alpha, 0)
$$

with $\lambda$ denotes the threshold used.

With this method, they used a threshold independent of the level and soft thresholding function whose expression is given by equation (10). Another approach to speech enhancement introduced by A. Sumithra MG et al [10] comprises applying to the noisy signal the Discrete Wavelet Transform (DWT) instead of the continuous wavelet transform discretized (CWT). The wavelet coefficients are then obtained multiplied by the factor $K(a, n)$ for the adaptation time. These coefficients are then thresholded using the soft thresholding function. The coefficients are then thresholded multiplied by a factor $\frac{1}{K(a, n)}$ and the inverse discrete wavelet transform is then applied to obtain the enhanced signal. This approach is summarized by the algorithm given in Figure 3[10]. 


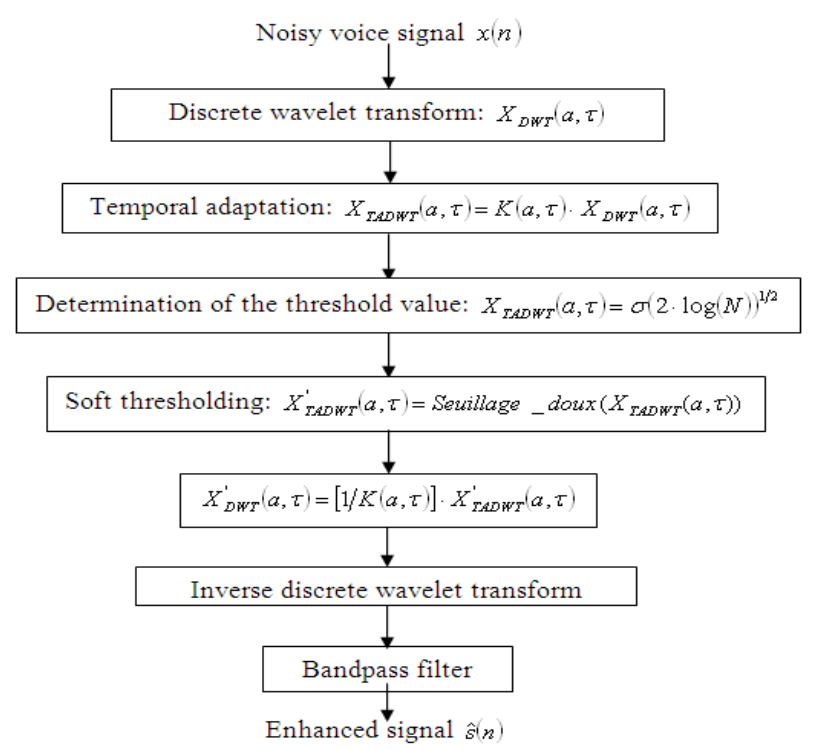

Figure 3. The block diagram of the proposed approach

\subsection{The Denoising by Wavelet}

The choice of the wavelet [13] can be tricky, but it is very important because it determines the quality of the results. This choice can be guided by:

$>$ A good compromise between time / frequency resolution,

$>$ Its mathematical properties: vanishing moments, regularity, size medium ...

A shape close to a pattern that you want to highlight in the signal without knowing the exact scale. Each type of mother wavelet has its own characteristics (shape, regularity, vanishing moments, time-frequency extension, etc). We therefore choose a particular analyzing wavelet depending on what you want to highlight in the signal. Orthogonal wavelets are wavelets first appeared in the work of Meyer and Mallat. A number of commonly used orthogonal families such as Daubechies wavelets. The non-orthogonal wavelet families are called wavelet biorthorthogonales. In fact, the prefix "bi" recalls that two wavelet bases are used, one is for the decomposition and reconstruction [14].

\subsubsection{The Haar Wavelet}

The first example of orthogonal wavelet is the Haar wavelet. It is fairly standard [14]. The mother wavelet is given by:

$$
\psi(x)=\left\{\begin{array}{l}
-1 \text { si } 0 \leq x \leq 0.5 \\
1 \text { si } 0.5<x \leq 1
\end{array}\right.
$$

\subsubsection{The Daubechies Wavelet}

These wavelets, especially suitable for multiresolution analysis, are not symmetrical and have a compact support [14]. Figure.5 represents the wavelet functions for different vanishing moments. There are other wavelets belonging to Daubechies system, the most important are: wavelets and wavelet symlets coiflets. 

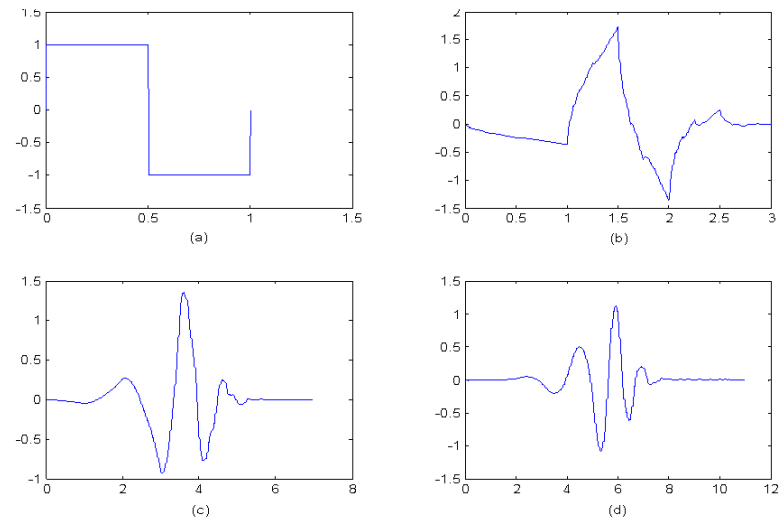

Figure 4. Examples wavelet (a) Haar wavelet, (b) Daubechies wavelet of order 2, (c) Daubechies wavelet of order 4, (d) Daubechies wavelet of order 6.

\section{THE CODING STRATEGY OF MULTI CHANNEL COCHLEAR IMPLANT}

Single electrode cochlear implant (CI) are failed mainly due to stimulate only a particular place in the cochlea due to the single electrode used. Thus single electrode CI can only provide very limited frequency information, since they use only one electrode and perform simple spectral analysis. To better exploit the place/frequency mechanism found in the peripheral auditory system, multi-channel CI were developed. Multi channel implants provide electrical stimulation at multiple sites in the cochlea using an array of electrodes. An electrode array is used so that different auditory nerve fibers can be stimulated at different places in the cochlea, thereby exploiting the place mechanism for coding frequencies. Different electrodes are stimulated depending on the frequency of the signal. Electrodes near the base of the cochlea are stimulated with high-frequency signals, while electrodes near the apex are stimulated with low-frequency signals.

The waveform based speech processing algorithms try to present some type of waveform (in analog or pulsatile form) derived from the speech signal by filtering into different frequency bands, while the feature-extraction based speech processing algorithms are try to present some type of spectral features, such as formants, derived using feature extraction algorithms. Hybrid algorithms presents the utilizing both algorithms. A brief coverage of these speech processing algorithms is given in Figure 5.Here the speech processor design using FPGA architecture system software comprises of two important modules, namely Programmable Speech Processing modules and Speech Data Encoding modules as shown in figure 6.

The Programmability of the speech processing system design described herein provides the means to develop and test 8 Channel Continuous Interleaved Sampled (CIS) speech processing algorithms. It provides flexibility and programmability according to patient's active electrodes. By using the impedance telemetry and clinical programming software, the audiologist identifies the active electrodes and sends this information to the Speech Processing module via Speech Data Encoding module of Xilinx, device FPGA Virtex5 XC5VLX110T card. [16]

The Band pass filters are configured so that the cutoff frequencies are adjusted depending on the number of active electrodes as shown in Table 1 and observe the block diagram of CIS algorithm in the figure 7.

The input signals are digitally filtered into 8 band-pass filters using Hamming window finite impulse response (FIR) filtering.. The input samples are stored in the circular buffer managed by 
Xilinx Virtex5 FPGA. Each input acoustic sample is fed to a bank of band-pass channels. Each channel includes the stages of band-pass filtering, envelope detection, compression and modulation.

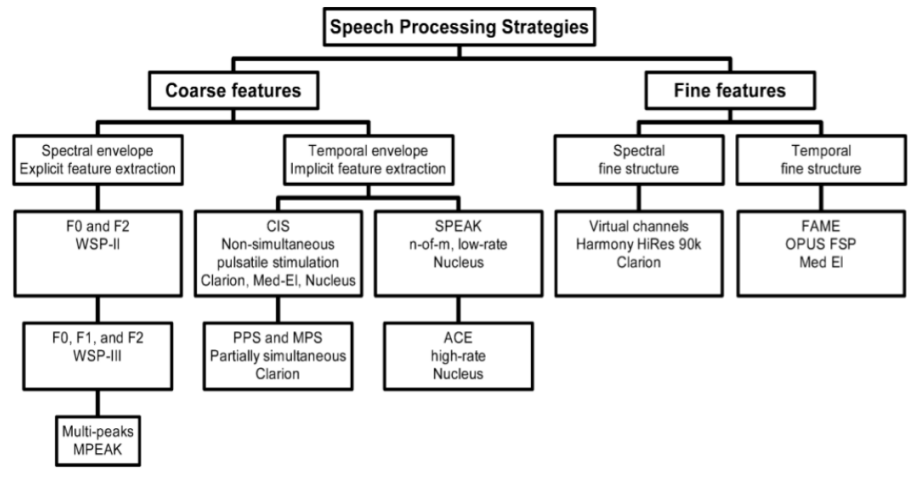

Figure 5. Classification coding strategies used in cochlear implants. [17-18].

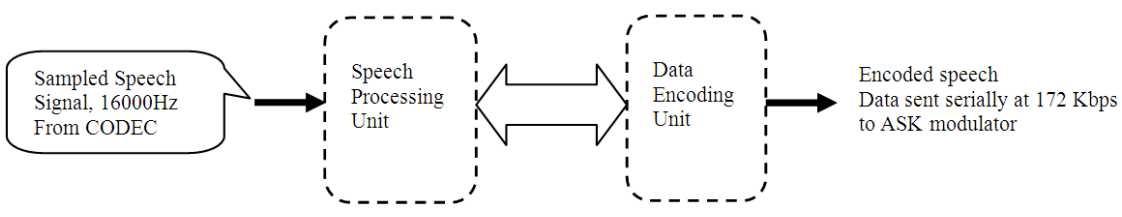

Figure 6. Functional Modules of the Speech Processing Design

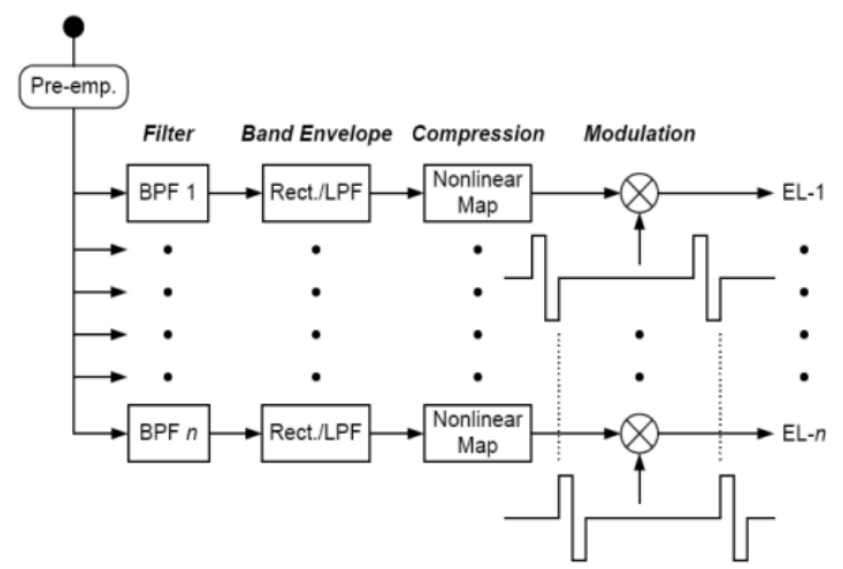

Figure 7. Block Diagram of CIS Algorithm

The temporal envelope in each channel is extracted with full-wave rectifier followed by $32 \mathrm{nd}$ order low pass Hamming window FIR filter. 
Table 1. Cut-off frequencies of Channels

\begin{tabular}{|c|c|c|c|}
\hline $\begin{array}{c}\text { Channe1 } \\
\text { number }\end{array}$ & $\begin{array}{c}\text { Lower } \\
\text { frequency }\end{array}$ & $\begin{array}{c}\text { Upper } \\
\text { frequency }\end{array}$ & $\begin{array}{c}\text { Center } \\
\text { frequency }\end{array}$ \\
\hline 1 & 200 & 310 & 255 \\
\hline 2 & 310 & 479 & 394 \\
\hline 3 & 479 & 742 & 611 \\
\hline 4 & 742 & 1149 & 946 \\
\hline 5 & 1149 & 1779 & 1464 \\
\hline 6 & 1779 & 2754 & 2266 \\
\hline 7 & 2754 & 4263 & 3508 \\
\hline 8 & 4263 & 6600 & 5432 \\
\hline
\end{tabular}

The low-pass filters were designed to smooth the amplitude estimates in time, and were set to a cutoff frequency of $200 \mathrm{~Hz}$ to allow maximal transmission of temporal envelope cues while avoiding aliasing when a relatively low carrier rates are used.

\subsection{Realization of FIR filters}

To test our filter, we use the method previously seen with the direct digital synthesis, The following program generates a signal whose frequency increases from $5 \mathrm{kHz}$ to $15 \mathrm{kHz}$ and 20 $\mathrm{kHz}$, all for a period of $3.14 \mu \mathrm{S}$. The program is written in VHDL and synthesized using the Xilinx ISE14.2 tool. Here is the diagram obtained for our filter Figure 8 :

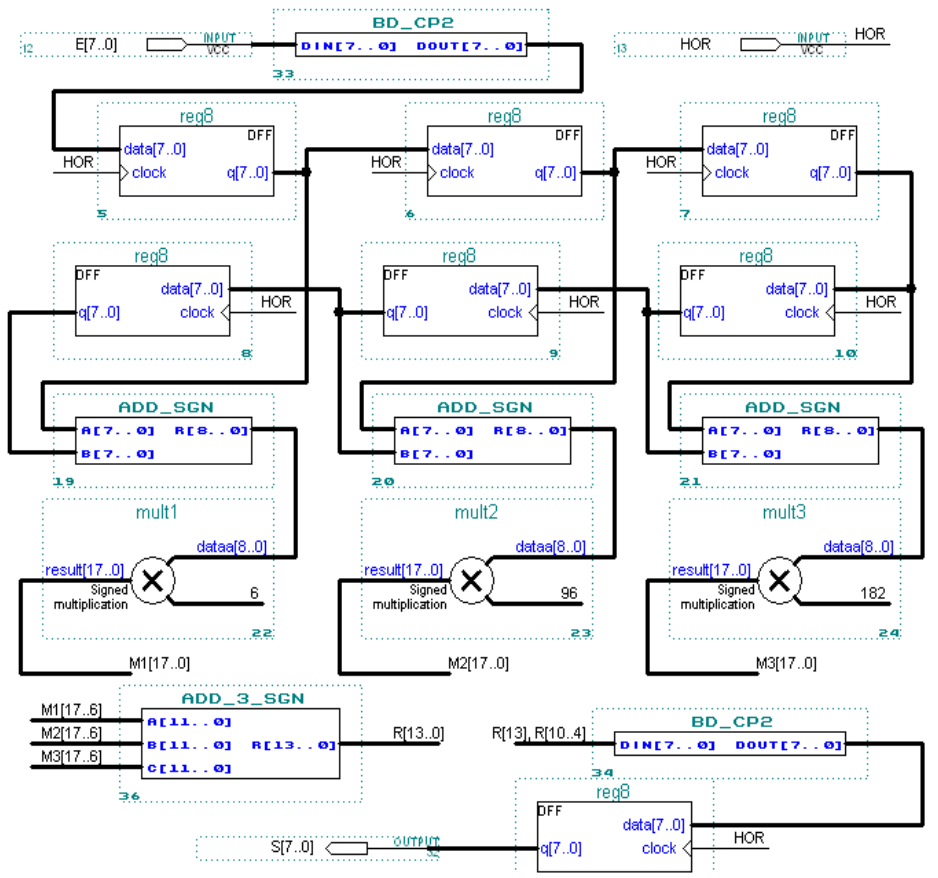

Figure 8. complete diagram of FIR filter in FPGA 
Using software ScopeFIR determining the coefficients of the low pass filter for a sampling frequency of $50 \mathrm{KHz}$, a band at $-3 \mathrm{~dB}$ from $5 \mathrm{KHz}$ and a $20 \mathrm{~dB}$ stopband $15 \mathrm{KHz}, 6$ coefficients of 9 bits. Then we get:

- $\mathrm{h}_{0}=\mathrm{h}_{5}=0.01171875_{\mathrm{D}}$

- $\mathrm{h}_{1}=\mathrm{h}_{4}=0.1875_{\mathrm{D}}$

- $\mathrm{h}_{2}=\mathrm{h}_{3}=0.35546875_{\mathrm{D}}$

and here is the output signal S[7..0] (Figure 9):

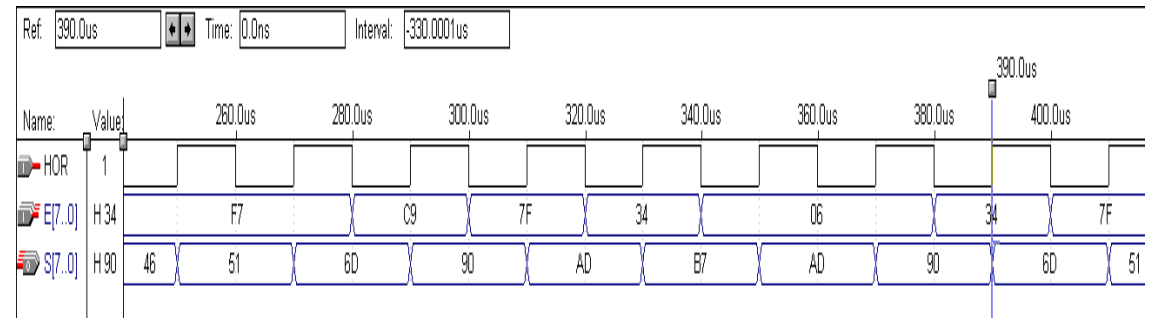

Figure 9.The output of the filtered signal

\section{The Main Design of Bionic Wavelet Transform in VHDL}

Figure 10 The following program, generates a signal whose frequency increases from $5 \mathrm{kHz}$, then to $15 \mathrm{kHz}$ to $20 \mathrm{kHz}$, all during a period of $3,14 \mu \mathrm{s}$. The program is set over four parties, the command block, the memory block, the operator block \& comparator block.

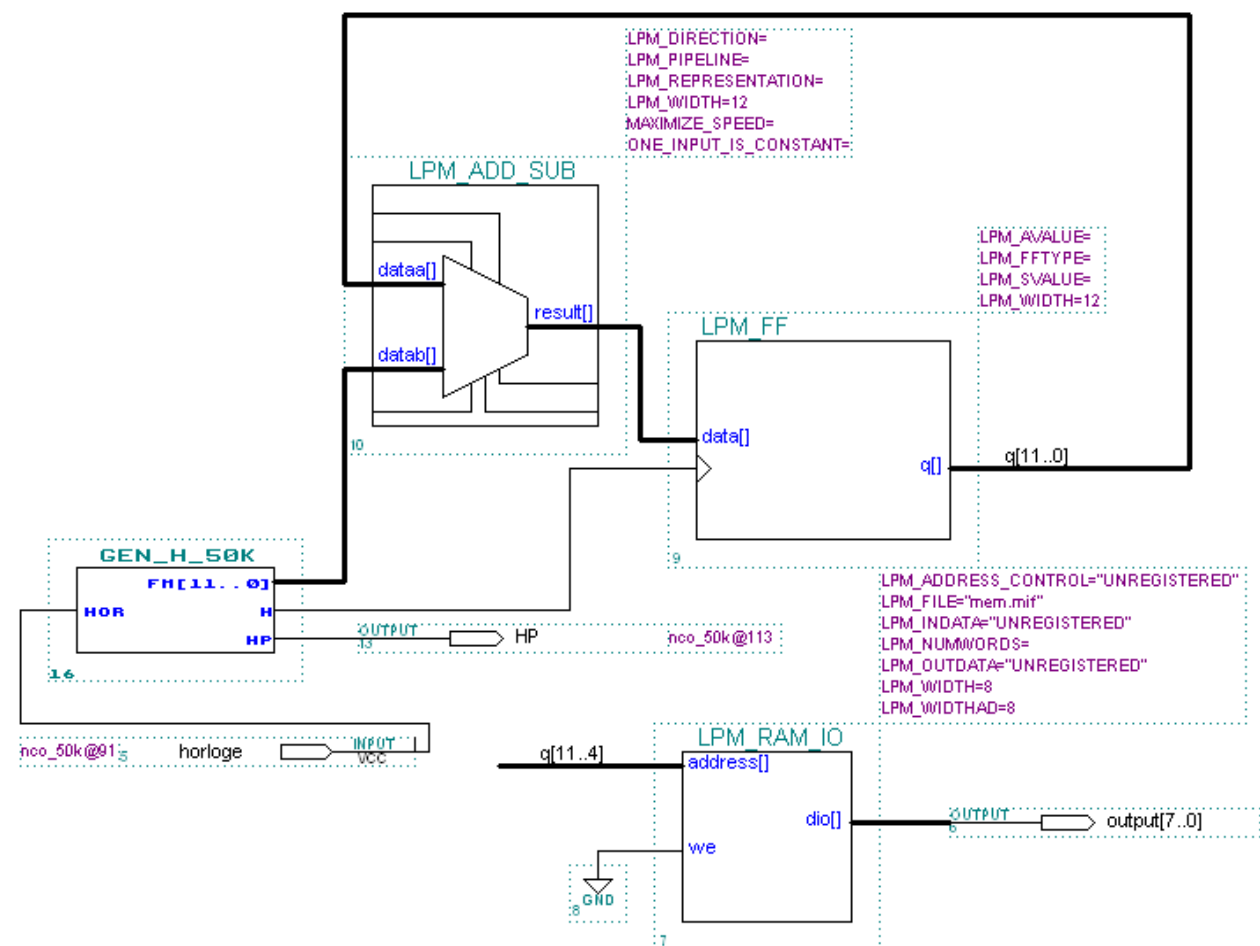

Figure 10. Complete diagram of BWT denoising in FPGA 


\section{SimUlation}

In this section we will present our approach to speech enhancement by thresholding both in the field of wavelet packet in the area of bionic wavelet transform (BWT).

\subsection{The wavelet packet tree used}

This method is to first apply the wavelet packet transform discrete DWPT followed by the cancellation of some terminal nodes of the tree of wavelet packet thresholding then obtained to remaining terminal nodes using function hard thresholding modified $\mu-l o i$ given by (11). [12]

$$
S(r, \lambda)=\left\{\begin{array}{c}
r \text { si }|r|>\lambda \\
\lambda\left(\frac{\left[(1+\mu)^{|r \lambda|}-1\right]}{\mu} \operatorname{sign}(r)\right) \text { si }|r|<\lambda
\end{array}\right.
$$

The elimination of these subbands is based on the fact that the speech signal is voiced consisting areas, areas unvoiced and quiet zones. Voiced sounds are quasi-periodic in the time domain and harmonically structured. In the frequency domain, they are usually located in the bands below 1 $\mathrm{KHz}$. However, the energy of unvoiced sounds is often concentrated at high frequencies $(\geq 3 \mathrm{KHz}$ ).If we want to distinguish between voiced sounds and unvoiced sounds, we should benefit from the information contained in these frequency bands where the sound is voiced or unvoiced dominant. It is well known that the power of the speech signal is contained around the first form.

The statistical results for several vowels of male and female voices, indicate approximately the frequency of the first formant does not exceed $1 \mathrm{KHz}$ and is not less than $100 \mathrm{KHz}$. In addition, the fundamental frequency of a normal voice, is between 80 and $500 \mathrm{~Hz}$. From here you can cancel the sub-bands which are less than $80 \mathrm{~Hz}$ and the remaining terminal nodes of the tree of wavelet packet obtained by applying the DWPT are subject to the thresholding operation by using the hard thresholding modified $\mu-l o i$. Speech signals used in the evaluation of this technique and other techniques are 20 sentences uttered by two Arabic speakers (ten sentences by a female voice and the other by a male voice). The sampling frequency is $16 \mathrm{KHz}$ and the treatment is carried out frame by frame. Each frame contains 512 samples with an overlap of 256 samples. The mother wavelet used in this method is the Daubechies wavelet, which has $40 \mathrm{~dB}$ greater perceptual property. The tree of wavelet packet employee is given in Figure 11. 


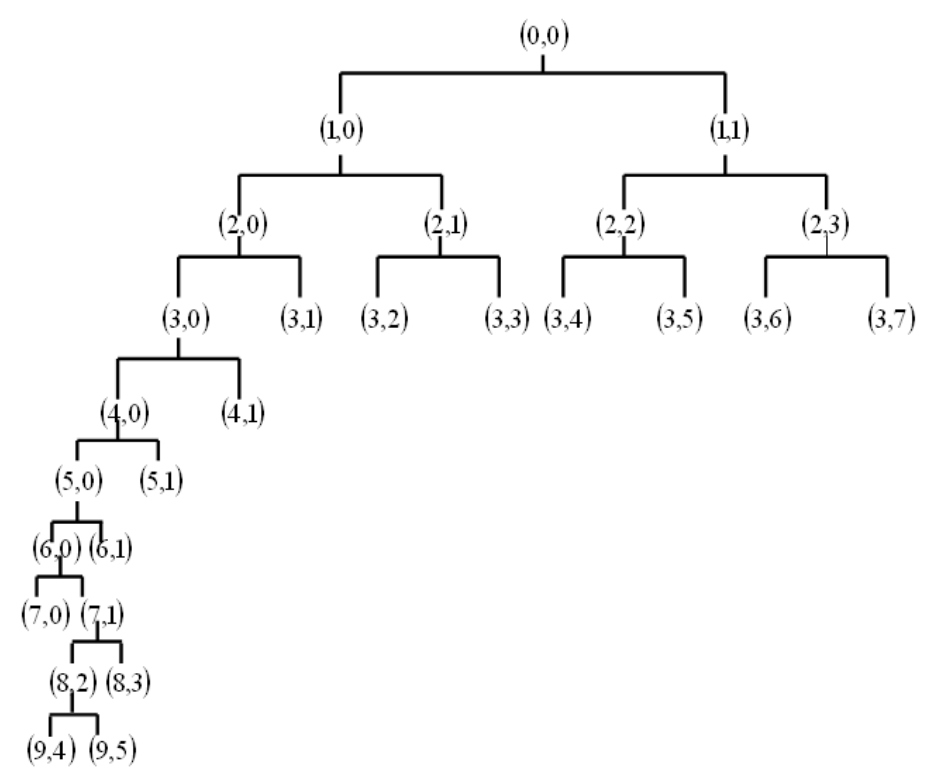

Figure 11. The tree of wavelet packet used

Nodes are set to zero in this tree are the nodes $(7,0)$ and $(9,4)$ because they occupy subbands that are lower than $80 \mathrm{~Hz}$. And these nodes are considered noise and are those things used to reestimate the noise level in the neighboring nodes: before the cancellation of the node $(7,0)$, it is used to estimate the noise level in the node $(7,1)$. The same applies to the node $(9,4)$ is employed to estimate the noise level in the node $(9,5)$, prior to its cancellation.

\subsection{Simulation and implementation in FPGA}

A test program was run on FPGA based speech processor with core frequency at $326 \mathrm{MHz}$ as the main processing unit, the ADC sampling rate at $64 \mathrm{kHz}$ and the encoding module of FPGA formatting and transmitting two data frames via the RF coil. A 128th order FIR program containing 8 band-pass filters runs on Xilinx Virtex5 FPGA processing unit. The input signal FPGA to the speech processor is received from the microphone.

Since the sampling rate is fixed at $64 \mathrm{KHz}$, we get 64000 samples for every second (i.e. 64 samples for every $1 \mathrm{~ms}$ ). These samples are fed to the filter bank, containing 8 band pass filters with frequencies ranging from $200 \mathrm{~Hz}$ to $6600 \mathrm{~Hz}$. Rectified signals are generated from each filter, which are then fed to the low pass filter with cutoff frequency of $200 \mathrm{~Hz}$ for envelope outputs. These 8 envelope signals are compressed using power law compression. These compressed signals of eight band-pass filters are transmitted to the encoding module in a base to apex order as shown in fig 10. This process is continuous and repeated for every $1.15 \mathrm{~ms}$. The FPGA based speech processor using Continuous Interleaved Sampling ( CIS) strategy $[6,7,8]$ has been run and tested with a laboratory model implant module.

and here is the output signal (Figure 12), (With the coefficients A and B of high and low frequency of transformation). 


\begin{tabular}{|c|c|c|c|c|c|c|c|c|c|c|c|}
\hline \multirow{2}{*}{$\begin{array}{l}\text { Ref: } 900.0 n s \\
\text { Name: }\end{array}$} & \multirow[b]{2}{*}{ Value: } & \multicolumn{2}{|c|}{ Time: $696.0 n s$} & \multirow{3}{*}{$\begin{array}{l}\text { Interval: }-104.0 \mathrm{nn} \\
\text { 200.0ns }\end{array}$} & \multirow[b]{2}{*}{ 300.0ns } & \multirow[b]{2}{*}{ 400.Ons } & \multirow[b]{2}{*}{ 500.Ons } & \multirow[b]{2}{*}{ 600.0ns } & \multirow[b]{2}{*}{ 700.0ns } & \multirow[b]{2}{*}{ 800.0ns } & \multirow{2}{*}{9000 Ons } \\
\hline & & & 100.0ns & & & & & & & & \\
\hline & 0 & & & & & & & & & & \\
\hline$=\mathrm{HP}$ & 1 & & & & & & & & & & \\
\hline$\exists=a[11.0]$ & HBE7 & $B A 2$ & $B A 3$ & BA1 & $B A D$ & BED & $\mathrm{BE} 1$ & $\mathrm{BE} 3$ & $\mathrm{BE} 2$ & BE6 & $\mathrm{BE} 7$ \\
\hline $\bar{\nabla} b[11.0]$ & HA.37 & A12 & A13 & A11 & $\mathrm{A10}$ & $A 30$ & $A 31$ & $A 33$ & A32 & $A 36$ & A37 \\
\hline 20 output & H 1584 & & & & & & $15 \mathrm{~B} 4$ & & & & \\
\hline$\equiv=7 \mid$ dataa $[12.0]$ & H 1 BE7 & $1 B A 2$ & $1 B A 3$ & $1 B A 1$ & 1BAD & $1 \mathrm{BED}$ & 1BE1 & 1BE3 & 1BE2 & 18E6 & 1BE7 \\
\hline $\bar{\nabla}=7 \mid$ datab[12.0] & H 1 A37 & $1 \mathrm{~A} 12$ & $1 \mathrm{~A} 13$ & $1 \mathrm{~A} 11$ & $1 \mathrm{~A} 10$ & $1 \mathrm{A30}$ & $1 A 31$ & 1 A33 & $1 A 32$ & $1 \mathrm{~A} 36$ & $1 A 37$ \\
\hline $\bar{\nabla}$ erldataa 12.0$]$ & H1BE7 & $1 B A 2$ & $1 B A 3$ & $1 \mathrm{BA1}$ & 1BAD & $1 \mathrm{BEO}$ & 1BE1 & $1 \mathrm{BE} 3$ & $1 \mathrm{BE} 2$ & 1BE6 & $1 \mathrm{BE} 7$ \\
\hline 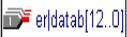 & H1A37 & $1 \mathrm{~A} 12$ & $1 \mathrm{~A} 13$ & $1 \mathrm{~A} 11$ & 1A10 & $1 \mathrm{A30}$ & $1 \mathrm{~A} 31$ & $1 A 33$ & $1 A 32$ & $1 \mathrm{A36}$ & $1 A 37$ \\
\hline
\end{tabular}

Figure 12. The output of the BWT module.

\section{RESULTS AND DISCUSSION}

Figures 13 (a), (b), (c) and (d) show that there is virtually no loss of information when we put the nodes $(7,0)$ and $(9,4)$ to zero .
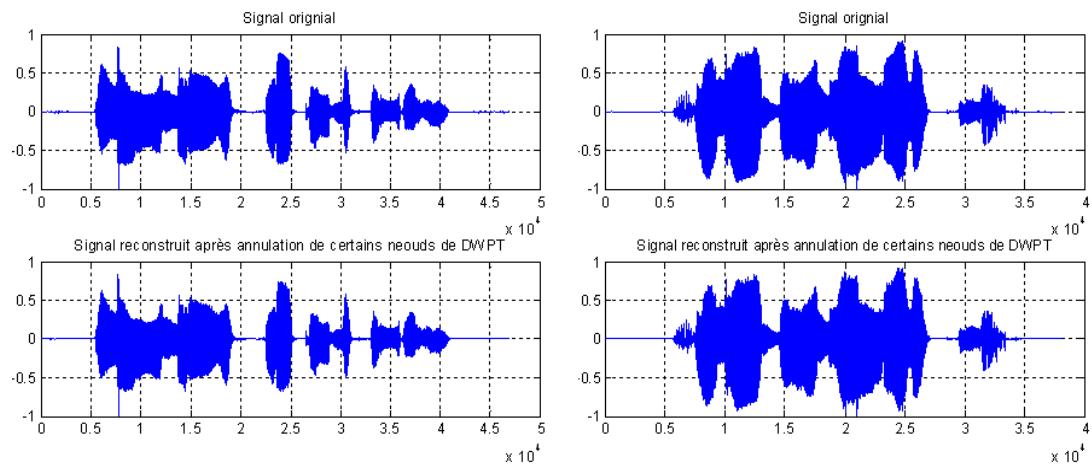

(a) A sample speech signal (Female Voice).

(b) A sample speech signal (Female Voice)
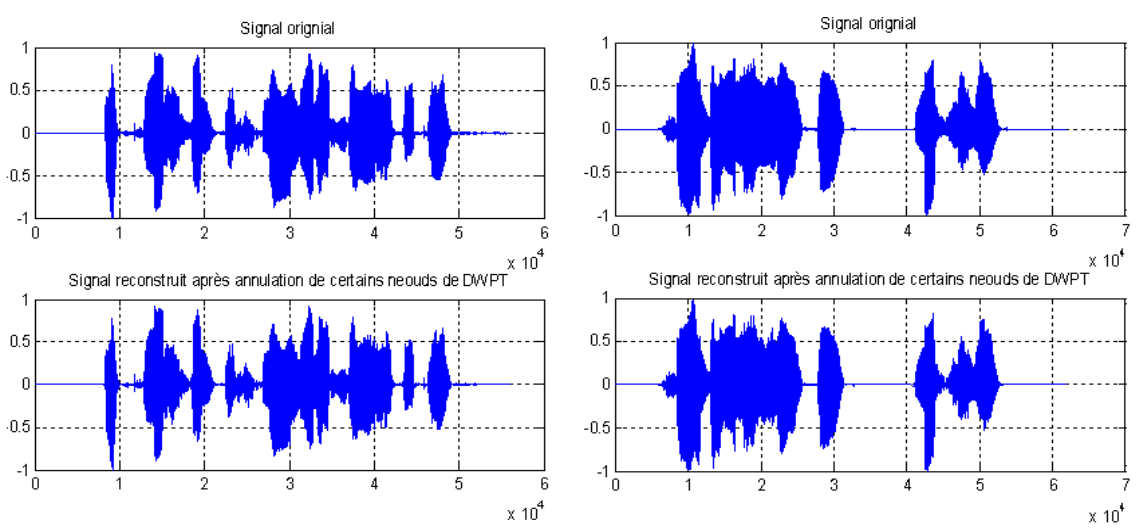

(c) A sample speech signal (Male Voice)

(d) A sample speech signal (Male Voice)

Figure 13. Perfect reconstruction after cancellation of nodes (7.0) and (9.4) 
Table 2 gives the values of SNRf (Signal to Noise Ratio after enhancement) obtained by applying the first method of denoising by thresholding and this for different values of (Signal to Noise Ratio before enhancement) SNRi $(-5,0,5,10$ and 15dB) and two types of broadband noise (white noise) and narrowband (Volvo car noise). For both male and female voices, SNRf values in this table 2 are average values.
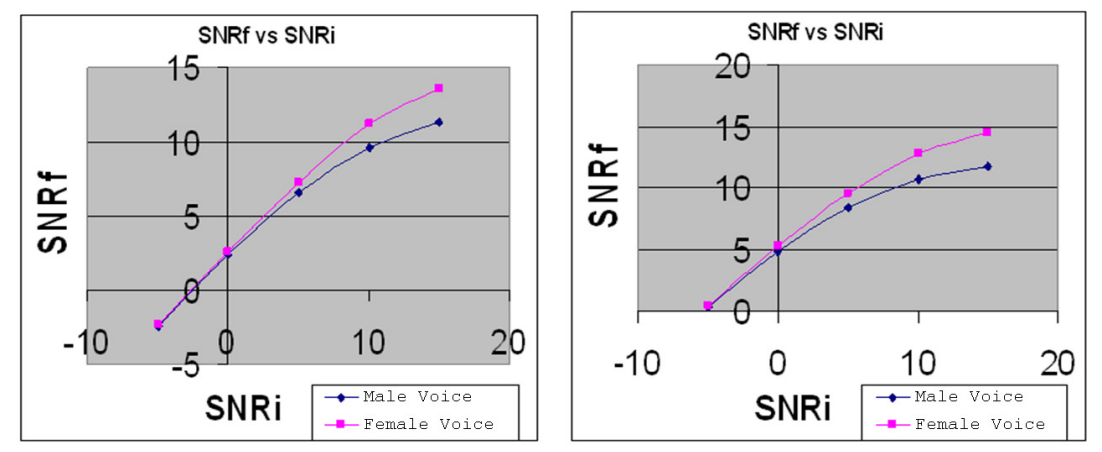

Figure 14. (a) Case of Volvo noise, (b) Cases of white noise

Table 2 and Figure 14 show that the first method improves the signal to noise ratio (SNRi $\leq$ $S N R f$ ). They also show that the results obtained in the case of female voices are better than those obtained in the case of the male voice.

Table 2. Average SNR calculated by applying the first thresholding method using modified thresholding function $\mu-l o i$.

\begin{tabular}{|c|c|c|c|c|}
\hline \multirow{2}{*}{ SNRi (dB) } & \multicolumn{4}{|c|}{ SNRf (dB) } \\
\cline { 2 - 5 } & \multicolumn{2}{|c|}{ male voice } & \multicolumn{2}{c|}{ female voice } \\
\cline { 2 - 5 } & $\begin{array}{c}\text { Volvo } \\
\text { noise }\end{array}$ & $\begin{array}{c}\text { white } \\
\text { noise }\end{array}$ & $\begin{array}{c}\text { Volvo } \\
\text { noise }\end{array}$ & $\begin{array}{c}\text { white } \\
\text { noise }\end{array}$ \\
\hline $\mathbf{- 5}$ & 0.36 & -2.46 & 0.50 & -2.37 \\
\hline $\mathbf{0}$ & 4.83 & 2.37 & 5.27 & 2.59 \\
\hline $\mathbf{5}$ & 8.44 & 6.60 & 9.52 & 7.21 \\
\hline $\mathbf{1 0}$ & 10.67 & 9.64 & 12.75 & 11.19 \\
\hline $\mathbf{1 5}$ & 11.69 & 11.29 & 14.43 & 13.56 \\
\hline
\end{tabular}

\section{CONCLUSIONS AND FUTURE DIRECTIONS}

In this paper, we proposed an approach to implementing one of the most used algorithms in cochlear implant via a development type Virtex5 FPGA card equipped with a chip of type XC5VLX110T. The main goal of this work is the enhancement of speech signal for the rehabilitation of deafness by cochlear implant. 
The advantage of this technique is that the mother wavelet analysis allows a shell variable with time and adapts according to the characteristics of the speech signal analysis, which improves the performance of the thresholding technique.

Although this success is encouraging, there is still much to learn about strategies electrical stimulation in the speech enhancement on cochlear implants, and many questions to be answered. In addition, reducing the number of channels with the intelligibility of the signal.

\section{REFERENCES}

[1] Yao J., "An active model for otoacoustic emissions and its application to time-frequency signal processing," PhD., The Chinese University of Hong Kong, Hong Kong, 2001.

[2] Yao J., Zhang, Y. T., "Bionic Wavelet Transform: a new time-frequency method based on auditory model,' IEEE Trans. Biomed. Eng., 48 (8), 856-863, 2001.

[3] Michael T. Johnson, Xiaolong Yuan, Yao Ren, "Speech signal enhancement through adaptive wavelet thresholding,' ScienceDirect, Speech communication 49 (2007) 123-133, 2007.

[4] Giguere C. Woodland P.C., "A computational model of the auditory periphery for speech and hearing research," J. Acoust. Soc. Amer. 95(1), 331-342, 1995.

[5] Xiaolong Yuan, B.S.E.E., “Auditory Model-based Bionic Wavelet Transform for Speech Enhancement," PhD thesis, MARQUETTE UNIVERSITY, Speech and Signal Processing Lab Milwaukee, Wisconsin, May 2003.

[6] C. Philipos LOIZOU "Speech Processing in Vocoder-Centric Cochlear Implants" Advances in Otorhinolaryngology Basel, Karger, vol. 64, p. 109-143,(2006).

[7] Fan-Gang Zeng, Stephen Rebscher William HARRISON, Xiaoan SUN \& FENG Haihong: "Cochlear Implants: System Design, Integration, and Evaluation" IEEE Reviews in Biomedical Engineering, vol. 1, p. 115-142, (2008).

[8] Ay, S., Zeng, F. -G. and Sheu, B. (1997). "Hearing with bionic ears: "Speech processing strategies for cochlear implant devices“, IEEE Circuits \& Devices Magazine, May 1997, 18-23.

[9] L. Breiman, "Better Subset Regression using the Non-negative Garrote,', Technometrics, vol. 37, pp. 327-384, 1995.

[10] A. Sumithra M G, B. Thanuskodi K, C. Anitha M R, "'Modified Time Adaptive Based Approach for Enhancing Speech from Adverse Noisy Environments,' DSP Journal of ICGST, Volume 9, Issue 1: 33-40. June 2009.

[11] Yao J., Zhang Y.T., "The application of bionic wavelet transform to speech signal processing in cochlear implants using neural network simulations', IEEE Trans. Biomed. Eng. 49 (11), 1299-1309, 2002.

[12] Talbi M., Salhi L., Chérif Adnane, "'Spectral entropy Employment in Speech Enhancement based on Wavelet Packet,' International Journal of Computer and Information Science and Engineering, pp.136-143, 2007.

[13] L'équipe technique Gresilog Traitement du signal, Bibliothèque ondelettes version1.0. http://www.Lis.inpg.fr/mustig/doc_html/ondlet/ondlet.htm.

[14] Morgan BRISHOUAL, "Reconstruction des données, application à la dosimétrie des Radiotéléphones', Thèse de doctorat, Electronique, L'Institut National des Sciences Appliquées de Rennes, Octobre 2001.

[15] D. Donho, I.M. Johnstone, “Adapting to unknown smoothness by wavelet shrinkage," Journal of the American statistical association, (90) 41, pp. 1200-1224, 1995.

[16] Site : Xilinx Platform FPGAs. "Available from": http://www.xilinx.com.

[17] C. Philipos LOIZOU: "Mimicking the Human Ear"IEEE Signal Processing Magazine, p. 101-130, (1998). 
Authors

Salaheddine DEROUICHE received his master in electrical engineering from UBMA University, ANNABA, Algeria in 2010, and searcher in LERICA laboratory at Annaba, Algeria since 2011. Currently, he is an assistant professor at Badji-Mokhtar University, Annaba, Algeria since 2011. His interests are in speech processing.

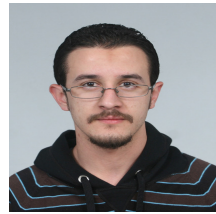

Djedou Bashir received his degree in Electronic Engineering in Algiers in 1978 and his doctorate in 1990 at the University Claude Bernard Lyon 1 - France. Currently, he is assistant professor at the University Badji Mokhtar, Annaba. His interests are in intelligent systems.

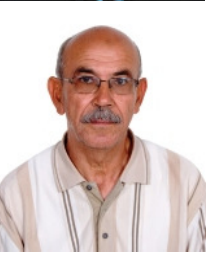

